\title{
FIXED POINTS OF OCCASIONALLY WEAKLY COMPATIBLE MAPPINGS USING IMPLICIT RELATION
}

\author{
Badri Datt Pant and Sunny Chauhan
}

\begin{abstract}
In this paper, we prove common fixed point theorems for families of occasionally weakly compatible mappings in Menger spaces using implicit relation. Our results extend and generalize the results of Altun and Turkoglu [9] in the sense that the concept of occasionally weakly compatible maps is the most general among all the commutativity concepts. Also the completeness of the whole space, continuity of the involved maps and containment of ranges amongst involved maps are completely relaxed.
\end{abstract}

\section{Introduction}

The concept of probabilistic metric space (in short PM-space) was first introduced and studied by Menger [28], which is a generalization of the metric space. The study of this space was expanded rapidly with the pioneering works of Schweizer and Sklar and some of their coworkers (see, [35], [36]). Such a probabilistic generalization of metric spaces appears to be well adapted for the investigation of physiological thresholds and physical quantities. It is also of fundamental importance in probabilistic functional analysis, nonlinear analysis and applications (see, [18], [22]). In 1972, Sehgal and Bharucha-Reid [37] initiated the study of contraction mappings in PM-spaces which is an important step in the development of fixed point theorems.

Jungck and Rhoades [24] introduced the notion of weakly compatible maps in metric spaces. Singh and Jain [39] formulated the notion of weakly compatible maps in probabilistic settings and proved some fixed point theorems in Menger spaces. In 2008, Al-Thagafi and Shahzad [7] introduced the notion of occasionally weakly compatible maps in metric spaces, while Chandra and Bhatt [17] extended the notion of occasionally weakly compatible maps in probabilistic setting. It is worth to mention that every pair of weak compatible self-maps is occasionally weak compatible but the reverse is not always true.

Received February 20, 2011; Revised June 24, 2011.

2010 Mathematics Subject Classification. Primary 47H10; Secondary 54H25.

Key words and phrases. triangle norm (t-norm), Menger space, occasionally weakly compatible maps, implicit relation, fixed point. 
Many authors proved common fixed point theorems using the notion of occasionally weakly compatible mappings on various spaces (see, [1]-[8], [10]-[17], [19]-[21], [25]-[27], [30], [34], [40]).

In 1998, Popa and Turkoglu [33] proved some fixed points theorem for hybrid mappings using the notion of implicit relation. Popa used the family of implicit real functions and proved some common fixed point theorems (see, [31], [32]). Recently, Altun and Turkoglu [9] proved two common fixed point theorems for continuous compatible mappings of type $(\alpha)$ and type $(\beta)$ on a complete fuzzy metric spaces with an implicit relation.

In the present paper, we prove common fixed point theorems for families of occasionally weakly compatible mappings in Menger spaces using implicit relation. Our results do not require completeness of the whole space, continuity of the involved maps and containment of ranges amongst involved maps. Also our results extend and generalize the results of Altun and Turkoglu [9] in many aspects.

\section{Preliminaries}

Definition 2.1 ([36]). A mapping $\triangle:[0,1] \times[0,1] \rightarrow[0,1]$ is t-norm if $\triangle$ is satisfying the following conditions:

(1) $\triangle$ is commutative and associative;

(2) $\triangle(a, 1)=a$ for all $a \in[0,1]$;

(3) $\triangle(a, b) \leq \triangle(c, d)$ whenever $a \leq c$ and $b \leq d$ and $a, b, c, d \in[0,1]$.

Examples of t-norms are $\triangle(a, b)=a \cdot b$ and $\triangle(a, b)=\min \{a, b\}$.

Definition 2.2 ([36]). A mapping $F: \mathbb{R} \rightarrow \mathbb{R}^{+}$is called a distribution function if it is non-decreasing and left continuous with $\inf \{F(t): t \in \mathbb{R}\}=0$ and $\sup \{F(t): t \in \mathbb{R}\}=1$.

We shall denote by $\Im$ the set of all distribution functions defined on $[-\infty, \infty]$ while $H(t)$ will always denote the specific distribution function defined by

$$
H(t)= \begin{cases}0, & \text { if } t \leq 0 \\ 1, & \text { if } t>0\end{cases}
$$

If $X$ is a non-empty set, $\mathcal{F}: X \times X \rightarrow \Im$ is called a probabilistic distance on $X$ and the value of $\mathcal{F}$ at $(x, y) \in X \times X$ is represented by $F_{x, y}$.

Definition 2.3 ([36]). A probabilistic metric space is an ordered pair $(X, \mathcal{F})$, where $X$ is a nonempty set of elements and $\mathcal{F}$ is a probabilistic distance satisfying the following conditions: for all $x, y, z \in X$ and $t, s>0$,

(1) $F_{x, y}(t)=H(t)$ for all $t>0$ if and only $x=y$;

(2) $F_{x, y}(0)=0$;

(3) $F_{x, y}(t)=F_{y, x}(t)$;

(4) if $F_{x, y}(t)=1$ and $F_{y, z}(s)=1$, then $F_{x, z}(t+s)=1$.

The ordered triple $(X, \mathcal{F}, \triangle)$ is called a Menger space if $(X, \mathcal{F})$ is a PMspace, $\triangle$ is a t-norm and the following inequality holds: 
(5) $F_{x, y}(t+s) \geq \triangle\left(F_{x, z}(t), F_{z, y}(s)\right)$ for all $x, y, z \in X$ and $t, s>0$.

Every metric space $(X, d)$ can always be realized as a PM-space by considering $\mathcal{F}: X \times X \rightarrow \Im$ defined by $F_{x, y}(t)=H(t-d(x, y))$ for all $x, y \in X$. So PM-spaces offer a wider framework than that of metric spaces and are better suited to cover even wider statistical situations.

Definition 2.4 ([39]). Self maps $A$ and $B$ of a Menger space $(X, \mathcal{F}, \triangle)$ are said to be weakly compatible (or coincidentally commuting) if they commute at their coincidence points, that is, if $A x=B x$ for some $x \in X$, then $A B x=B A x$.

The concept of occasionally weakly compatible maps due to [7] is a proper generalization of nontrivial weakly compatible maps which do have a coincidence point. The counterpart of the concept of occasionally weakly compatible maps in PM-spaces is as follows:

Definition 2.5. Two self maps $A$ and $B$ of a Menger space $(X, \mathcal{F}, \triangle)$ are occasionally weakly compatible if and only if there is a point $x \in X$ which is a coincidence point of $A$ and $B$ at which $A$ and $B$ commute.

From the following example it is clear that the notion of occasionally weakly compatible is more general than weak compatibility.

Example 2.6. Let $(X, \mathcal{F}, \triangle)$ be a Menger space, where $X=\mathbb{R}$ and

$$
F_{x, y}(t)= \begin{cases}\frac{t}{t+|x-y|}, & \text { if } t>0 \\ 0, & \text { if } t=0\end{cases}
$$

Define $A, B: \mathbb{R} \rightarrow \mathbb{R}$ by $A x=3 x$ and $B x=x^{2}$ for all $x \in \mathbb{R}$. Then $A x=B x$ for $x=0,3$ but $A B(0)=B A(0)$, and $A B(3) \neq B A(3)$. Thus $A$ and $B$ are occasionally weakly compatible maps but not weakly compatible.

Lemma 2.7. Let $(X, \mathcal{F}, \triangle)$ be a Menger space, $A$ and $B$ be occasionally weakly compatible self maps of $X$. If $A$ and $B$ have a unique point of coincidence, $w=A x=B x$, then $w$ is the unique common fixed point of $A$ and $B$.

Proof. Since $A$ and $B$ are occasionally weakly compatible, there exists a point $x$ in $X$ such that $A x=B x=w$ and $A B x=B A x$. Thus, $A A x=A B x=B A x$, which says that $A x$ is also a point of coincidence of $A$ and $B$. Since the point of coincidence $w=A x$ is unique by hypothesis, $B A x=A A x=A x$, and $w=A x$ is a common fixed point of $A$ and $B$.

Moreover, if $z$ is any common fixed point of $A$ and $B$, then $z=A z=B z=w$ by the uniqueness of the point of coincidence.

Lemma $2.8([29])$. Let $(X, \mathcal{F}, \triangle)$ be a Menger space. If there exists a constant $k \in(0,1)$ such that

$$
F_{x, y}(k t) \geq F_{x, y}(t)
$$

for all $t>0$ with fixed $x, y \in X$, then $x=y$. 


\section{Implicit relation}

Many authors proved a number of common fixed point theorems using the notion of implicit relation on different spaces (see [9], [23], [31]-[33], [38]).

Let $I=[0,1], \triangle$ be a continuous t-norm and $\varphi: I^{6} \rightarrow \mathbb{R}$ be a continuous function. Now, we consider the following conditions:

$(\varphi-1) \varphi$ is non-increasing in the fifth and sixth variables,

$(\varphi$-2) If, for some constant $k \in(0,1)$, we have

or

$$
\left(\varphi_{a}\right) \quad \varphi\left(u(k t), v(t), v(t), u(t), 1, \triangle\left(u\left(\frac{t}{2}\right), v\left(\frac{t}{2}\right)\right)\right) \geq 1,
$$$$
\left(\varphi_{b}\right) \quad \varphi\left(u(k t), v(t), u(t), v(t), \triangle\left(u\left(\frac{t}{2}\right), v\left(\frac{t}{2}\right)\right), 1\right) \geq 1
$$

for any fixed $t>0$ and any non-decreasing functions $u, v:(0, \infty) \rightarrow I$ with $0<u(t), v(t) \leq 1$, then there exists $h \in(0,1)$ with $u(h t) \geq \triangle(v(t), u(t))$.

$(\varphi-3)$ If, for some constant $k \in(0,1)$, we have

$$
\varphi(u(k t), u(t), 1,1, u(t), u(t)) \geq 1
$$

for any fixed $t>0$ and any non-decreasing function $u:(0, \infty) \rightarrow I$, then $u(k t) \geq u(t)$.

Now, let $\Phi$ be the set of all real continuous functions $\varphi: I^{6} \rightarrow \mathbb{R}$ satisfying the conditions $(\varphi-1) \sim(\varphi-3)$.

Example 3.1 $([9])$. Let $\varphi\left(u_{1}, \ldots, u_{6}\right)=\frac{u_{1}}{\min \left\{u_{2}, \ldots, u_{6}\right\}}$ and $\triangle(a, b)=\min \{a, b\}$.

Let $t>0,0<u(t), v(t) \leq 1, k \in\left(0, \frac{1}{2}\right)$, where $u, v:[0, \infty) \rightarrow I$ are nondecreasing functions. Now, suppose that

$$
\varphi\left(u(k t), v(t), v(t), u(t), 1, \triangle\left(u\left(\frac{t}{2}\right), v\left(\frac{t}{2}\right)\right)\right) \geq 1,
$$

i.e.,

$$
\begin{aligned}
\varphi\left(u(k t), v(t), v(t), u(t), 1, \triangle\left(u\left(\frac{t}{2}\right), v\left(\frac{t}{2}\right)\right)\right) & =\frac{u(k t)}{\min \left\{v(t), u(t), 1, \triangle\left(u\left(\frac{t}{2}\right), v\left(\frac{t}{2}\right)\right)\right\}} \\
& =\frac{u(k t)}{\min \left\{v\left(\frac{t}{2}\right), u\left(\frac{t}{2}\right)\right\}} \geq 1 .
\end{aligned}
$$

Thus $u(h t) \geq \triangle(v(t), u(t))$ if $h=2 k \in(0,1)$. A similar argument works if $\left(\varphi_{b}\right)$ is assumed. Finally, suppose that $t>0$ is fixed, $u:(0, \infty) \rightarrow I$ is a non-decreasing function and

$$
\varphi(u(k t), u(t), 1,1, u(t), u(t))=\frac{u(k t)}{u(t)} \geq 1
$$

for some $k \in(0,1)$. Then we have $u(k t) \geq u(t)$ and thus $\varphi \in \Phi$.

\section{Results}

In this section, first we prove a common fixed point theorem for any even number of occasionally weakly compatible maps in Menger space using implicit relation.

Theorem 4.1. Let $P_{1}, P_{2}, \ldots, P_{2 n}, A$ and $B$ be self maps on a Menger space $(X, \mathcal{F}, \triangle)$ where $\triangle$ is a continuous t-norm satisfying the following conditions: 
(4.1) there exists $k \in(0,1)$ and $\varphi \in \Phi$ such that

$$
\varphi\left(\begin{array}{c}
F_{A x, B y}(k t), F_{P_{1} P_{3} \cdots P_{2 n-1} x, P_{2} P_{4} \cdots P_{2 n} y}(t), F_{A x, P_{1} P_{3} \cdots P_{2 n-1} x}(t), \\
F_{B y, P_{2} P_{4} \cdots P_{2 n} y}(t), F_{A x, P_{2} P_{4} \cdots P_{2 n} y}(t), F_{B y, P_{1} P_{3} \cdots P_{2 n-1} x}(t)
\end{array}\right) \geq 1
$$

for all $x, y \in X$ and $t>0$.

Suppose that

$$
\begin{aligned}
P_{1}\left(P_{3} \cdots P_{2 n-1}\right) & =\left(P_{3} \cdots P_{2 n-1}\right) P_{1}, \\
P_{1} P_{3}\left(P_{5} \cdots P_{2 n-1}\right) & =\left(P_{5} \cdots P_{2 n-1}\right) P_{1} P_{3}, \\
& \vdots \\
P_{1} \cdots P_{2 n-3}\left(P_{2 n-1}\right) & =\left(P_{2 n-1}\right) P_{1} \cdots P_{2 n-3}, \\
A\left(P_{3} \cdots P_{2 n-1}\right) & =\left(P_{3} \cdots P_{2 n-1}\right) A, \\
A\left(P_{5} \cdots P_{2 n-1}\right) & =\left(P_{5} \cdots P_{2 n-1}\right) A, \\
& \vdots \\
A P_{2 n-1} & =P_{2 n-1} A,
\end{aligned}
$$

similarly,

$$
\begin{aligned}
P_{2}\left(P_{4} \cdots P_{2 n}\right) & =\left(P_{4} \cdots P_{2 n}\right) P_{2}, \\
P_{2} P_{4}\left(P_{6} \cdots P_{2 n}\right) & =\left(P_{6} \cdots P_{2 n}\right) P_{2} P_{4}, \\
& \vdots \\
P_{2} \cdots P_{2 n-2}\left(P_{2 n}\right) & =\left(P_{2 n}\right) P_{2} \cdots P_{2 n-2}, \\
B\left(P_{4} \cdots P_{2 n}\right) & =\left(P_{4} \cdots P_{2 n}\right) B, \\
B\left(P_{6} \cdots P_{2 n}\right) & =\left(P_{6} \cdots P_{2 n}\right) B, \\
& \vdots \\
B P_{2 n} & =P_{2 n} B .
\end{aligned}
$$

Then, if the pairs $\left\{A, P_{1} P_{3} \cdots P_{2 n-1}\right\}$ and $\left\{B, P_{2} P_{4} \cdots P_{2 n}\right\}$ are occasionally weakly compatible, it follows that $P_{1}, P_{2}, \ldots, P_{2 n}, A$ and $B$ have a unique common fixed in $X$.

Proof. Since the pairs $\left\{A, P_{1} P_{3} \cdots P_{2 n-1}\right\}$ and $\left\{B, P_{2} P_{4} \cdots P_{2 n}\right\}$ are occasionally weakly compatible then there exist points $u, v \in X$ such that $A u=$ $P_{1} P_{3} \cdots P_{2 n-1} u, A\left(P_{1} P_{3} \cdots P_{2 n-1}\right) u=\left(P_{1} P_{3} \cdots P_{2 n-1}\right) A u$ and $B v=P_{2} P_{4} \cdots$ $P_{2 n} v, B\left(P_{2} P_{4} \cdots P_{2 n}\right) v=\left(P_{2} P_{4} \cdots P_{2 n}\right) B v$. Now we show that $A u=B v$. Put $x=u$ and $y=v$ in the inequality (4.1), then we get

$$
\begin{aligned}
\varphi\left(\begin{array}{c}
F_{A u, B v}(k t), F_{P_{1} P_{3} \cdots P_{2 n-1} u, P_{2} P_{4} \cdots P_{2 n} v}(t), F_{A u, P_{1} P_{3} \cdots P_{2 n-1} u}(t), \\
F_{B v, P_{2} P_{4} \cdots P_{2 n} v}(t), F_{A u, P_{2} P_{4} \cdots P_{2 n} v}(t), F_{B v, P_{1} P_{3} \cdots P_{2 n-1} u}(t)
\end{array}\right) & \geq 1, \\
\varphi\left(F_{A u, B v}(k t), F_{A u, B v}(t), F_{A u, A u}(t), F_{B v, B v}(t), F_{A u, B v}(t), F_{B v, A u}(t)\right) & \geq 1, \\
\varphi\left(F_{A u, B v}(k t), F_{A u, B v}(t), 1,1, F_{A u, B v}(t), F_{B v, A u}(t)\right) & \geq 1 .
\end{aligned}
$$


Thus, from $(\varphi-3)$, we have

$$
F_{A u, B v}(k t) \geq F_{A u, B v}(t) .
$$

From Lemma 2.8, we have $A u=B v$. Moreover, if there is another point $z$ such that $A z=\left(P_{1} P_{3} \cdots P_{2 n-1}\right) z$. Then using the inequality (4.1) it follows that $A z=\left(P_{1} P_{3} \cdots P_{2 n-1}\right) z=B v=\left(P_{2} P_{4} \cdots P_{2 n}\right) v$, or $A u=A z$. Hence $w=A u=\left(P_{1} P_{3} \cdots P_{2 n-1}\right) u$ is the unique point of coincidence of $A$ and $P_{1} P_{3} \cdots P_{2 n-1}$. By Lemma 2.7, it follows that $w$ is the unique common fixed point of $A$ and $P_{1} P_{3} \cdots P_{2 n-1}$. By symmetry, $q=B v=\left(P_{2} P_{4} \cdots P_{2 n}\right) v$ is the unique common fixed point of $B$ and $P_{2} P_{4} \cdots P_{2 n}$. Since $w=q$, we obtain that $w$ is the unique common fixed point of $B$ and $P_{2} P_{4} \cdots P_{2 n}$. Now we show that $w$ is the fixed point of all the component mappings. Taking $x=\left(P_{3} \cdots P_{2 n-1}\right) w$, $y=w, P_{1}^{\prime}=P_{1} P_{3} \cdots P_{2 n-1}$ and $P_{2}^{\prime}=P_{2} P_{4} \cdots P_{2 n}$ in the inequality (4.1), we have

$$
\begin{gathered}
\varphi\left(\begin{array}{c}
F_{A P_{3} \cdots P_{2 n-1} w, B w}(k t), F_{P_{1}^{\prime} P_{3} \cdots P_{2 n-1} w, P_{2}^{\prime} w}(t), \\
F_{A P_{3} \cdots P_{2 n-1} w, P_{1}^{\prime} P_{3} \cdots P_{2 n-1} w}(t), F_{B w, P_{2}^{\prime} w}(t), \\
F_{A P_{3} \cdots P_{2 n-1} w, P_{2}^{\prime} w}(t), F_{B w, P_{1}^{\prime} P_{3} \cdots P_{2 n-1} w}(t)
\end{array}\right) \geq 1, \\
\varphi\left(\begin{array}{c}
F_{P_{3} \cdots P_{2 n-1} w, w}(k t), F_{P_{3} \cdots P_{2 n-1} w, w}(t), \\
F_{P_{3} \cdots P_{2 n-1} w, P_{3} \cdots P_{2 n-1} w}(t), F_{w, w}(t), \\
F_{P_{3} \cdots P_{2 n-1} w, w}(t), F_{w, P_{3} \cdots P_{2 n-1} w}(t)
\end{array}\right) \geq 1, \\
\varphi\left(\begin{array}{c}
F_{P_{3} \cdots P_{2 n-1} w, w}(k t), F_{P_{3} \cdots P_{2 n-1} w, w}(t), 1,1, \\
F_{P_{3} \cdots P_{2 n-1} w, w}(t), F_{w, P_{3} \cdots P_{2 n-1} w}(t)
\end{array}\right) \geq 1 .
\end{gathered}
$$

Thus, from $(\varphi-3)$, we have

$$
F_{P_{3} \cdots P_{2 n-1} w, w}(k t) \geq F_{P_{3} \cdots P_{2 n-1} w, w}(t) .
$$

From Lemma 2.8, we get $P_{3} \cdots P_{2 n-1} w=w$. Hence, $P_{1} w=w$. Continuing this procedure, we have

$$
A w=P_{1} w=P_{3} w=\cdots=P_{2 n-1} w=w .
$$

So,

$$
B w=P_{2} w=P_{4} w=\cdots=P_{2 n} w=w .
$$

Therefore, $w$ is the unique common fixed point of $P_{1}, P_{2}, \ldots, P_{2 n}, A$ and $B$.

The following result is a slight generalization of Theorem 4.1.

Theorem 4.2. Let $\left\{T_{\alpha}\right\}_{\alpha \in J}$ and $\left\{P_{i}\right\}_{i=1}^{2 n}$ be two families of self mappings on a Menger space $(X, \mathcal{F}, \triangle)$ where $\triangle i$ s a continuous -norm satisfying the following conditions:

(4.2) there exists a fixed $\beta \in J, k \in(0,1)$ and $\varphi \in \Phi$ such that

$$
\varphi\left(\begin{array}{c}
F_{T_{\alpha} x, T_{\beta} y}(k t), F_{P_{1} P_{3} \ldots P_{2 n-1} x, P_{2} P_{4} \ldots P_{2 n} y}(t), F_{T_{\alpha} x, P_{1} P_{3} \ldots P_{2 n-1} x}(t), \\
F_{T_{\beta} y, P_{2} P_{4} \ldots P_{2 n} y}(t), F_{T_{\alpha} x, P_{2} P_{4} \ldots P_{2 n} y}(t), F_{T_{\beta} y, P_{1} P_{3} \ldots P_{2 n-1} x}(t)
\end{array}\right) \geq 1
$$

for all $x, y \in X$ and $t>0$. 
Suppose that

$$
\begin{aligned}
P_{1}\left(P_{3} \cdots P_{2 n-1}\right) & =\left(P_{3} \cdots P_{2 n-1}\right) P_{1}, \\
P_{1} P_{3}\left(P_{5} \cdots P_{2 n-1}\right) & =\left(P_{5} \cdots P_{2 n-1}\right) P_{1} P_{3}, \\
& \vdots \\
P_{1} \cdots P_{2 n-3}\left(P_{2 n-1}\right) & =\left(P_{2 n-1}\right) P_{1} \cdots P_{2 n-3}, \\
T_{\alpha}\left(P_{3} \cdots P_{2 n-1}\right) & =\left(P_{3} \cdots P_{2 n-1}\right) T_{\alpha}, \\
T_{\alpha}\left(P_{5} \cdots P_{2 n-1}\right) & =\left(P_{5} \cdots P_{2 n-1}\right) T_{\alpha}, \\
& \vdots \\
T_{\alpha} P_{2 n-1} & =P_{2 n-1} T_{\alpha},
\end{aligned}
$$

similarly,

$$
\begin{aligned}
P_{2}\left(P_{4} \cdots P_{2 n}\right) & =\left(P_{4} \cdots P_{2 n}\right) P_{2}, \\
P_{2} P_{4}\left(P_{6} \cdots P_{2 n}\right)= & \left(P_{6} \cdots P_{2 n}\right) P_{2} P_{4}, \\
& \vdots \\
P_{2} \cdots P_{2 n-2}\left(P_{2 n}\right) & =\left(P_{2 n}\right) P_{2} \cdots P_{2 n-2}, \\
T_{\beta}\left(P_{4} \cdots P_{2 n}\right)= & \left(P_{4} \cdots P_{2 n}\right) T_{\beta}, \\
T_{\beta}\left(P_{6} \cdots P_{2 n}\right)= & \left(P_{6} \cdots P_{2 n}\right) T_{\beta}, \\
& \vdots \\
T_{\beta} P_{2 n} & =P_{2 n} T_{\beta} .
\end{aligned}
$$

Then, if the pairs $\left\{T_{\alpha}, P_{1} P_{3} \cdots P_{2 n-1}\right\}$ and $\left\{T_{\beta}, P_{2} P_{4} \cdots P_{2 n}\right\}$ are occasionally weakly compatible, it follows that all $\left\{P_{i}\right\}$ and $\left\{T_{\alpha}\right\}$ have a unique common fixed in $X$.

Proof. Since the proof is straightforward, we omit it.

Corollary 4.3. Let $A, B, S$ and $T$ be self maps on a Menger space $(X, \mathcal{F}, \triangle)$ where $\triangle$ is a continuous -norm satisfying the following conditions:

(4.3) there exists $k \in(0,1)$ and $\varphi \in \Phi$ such that

$$
\varphi\left(F_{A x, B y}(k t), F_{S x, T y}(t), F_{A x, S x}(t), F_{B y, T y}(t), F_{A x, T y}(t), F_{B y, S x}(t)\right) \geq 1
$$

for all $x, y \in X$ and $t>0$. Then, if the pairs $\{A, S\}$ and $\{B, T\}$ are occasionally weakly compatible, there exists a unique point $w \in X$ such that $A w=S w=w$ and a unique point $z \in X$ such that $B z=T z=z$. Moreover, $z=w$, so that there is a unique common fixed point of $A, B, S$ and $T$ in $X$.

Proof. If we set $P_{1} P_{3} \cdots P_{2 n-1}=S$ and $P_{2} P_{4} \cdots P_{2 n}=T$ in Theorem 4.1 , then the result follows.

Now, we give an example which illustrates Corollary 4.3. 
Example 4.4. Let $X=[0,2]$ with the metric $d$ defined by $d(x, y)=|x-y|$ and for each $t \in[0,1]$, define

$$
F_{x, y}(t)= \begin{cases}\frac{t}{t+|x-y|}, & \text { if } t>0 \\ 0, & \text { if } t=0\end{cases}
$$

for all $x, y \in X$. Clearly $(X, \mathcal{F}, \triangle)$ is a Menger space, where $\triangle$ is a continuous t-norm. Let $\varphi: I^{6} \rightarrow \mathbb{R}$ be defined as in Example 3.1 and define the self maps $A, B, S$ and $T$ by

$$
\begin{aligned}
& A(x)=\left\{\begin{array}{ll}
x, & \text { if } 0 \leq x \leq 1 ; \\
2, & \text { if } 1<x \leq 2 .
\end{array} \quad S(x)= \begin{cases}1, & \text { if } 0 \leq x \leq 1 ; \\
0, & \text { if } 1<x \leq 2\end{cases} \right. \\
& B(x)=\left\{\begin{array}{ll}
1, & \text { if } 0 \leq x \leq 1 ; \\
2, & \text { if } 1<x \leq 2 .
\end{array} \quad T(x)= \begin{cases}1, & \text { if } 0 \leq x \leq 1 ; \\
\frac{x}{2}, & \text { if } 1<x \leq 2\end{cases} \right.
\end{aligned}
$$

Then $A, B, S$ and $T$ satisfy all the conditions of Corollary 4.3 for some $k \in(0,1)$ with respect to the distribution function $F_{x, y}$.

First, we have

$$
A(1)=1=S(1) \text { and } A S(1)=1=S A(1),
$$

and

$$
B(1)=1=T(1) \text { and } B T(1)=1=T B(1) .
$$

That is, $A$ and $S$ as well as $B$ and $T$ are occasionally weakly compatible maps. Also 1 is the unique common fixed point of $A, B, S$ and $T$. On the other hand, it is clear to see that the maps $A, B, S$ and $T$ are discontinuous at 1.

On taking $A=B$ and $S=T$ in Corollary 4.3, we get the following result.

Corollary 4.5. Let $A$ and $S$ be self maps on a Menger space $(X, \mathcal{F}, \triangle)$ where $\triangle$ is a continuous $t$-norm satisfying the following conditions:

(4.4) there exists $k \in(0,1)$ and $\varphi \in \Phi$ such that

$$
\varphi\left(F_{A x, A y}(k t), F_{S x, S y}(t), F_{A x, S x}(t), F_{A y, S y}(t), F_{A x, S y}(t), F_{A y, S x}(t)\right) \geq 1
$$

for all $x, y \in X$ and $t>0$. Then, if the pair $\{A, S\}$ is occasionally weakly compatible, it follows that $A$ and $S$ have a unique common fixed in $X$.

Remark 4.6. Our results extend and generalize the results of Altun and Turkoglu ([9], Theorem 1 and Theorem 2) in the sense that the concept of occasionally weakly compatible maps is the most general among all the commutativity concepts. Also the completeness of the whole space, continuity of the involved maps and containment of ranges amongst involved maps are completely relaxed.

Acknowledgements. Authors would like to express their sincere thanks to Prof. Calogero Vetro for his papers [21], [40] and the referee for his valuable suggestions to improve the paper. 


\section{References}

[1] C. T. Aage and J. N. Salunke, On fixed point theorems in fuzzy metric spaces, Int. J. Open Probl. Comput. Sci. Math. 3 (2010), no. 2, 123-131.

[2] M. Abbas and B. E. Rhoades, Common fixed point theorems for hybrid pairs of occasionally weakly compatible mappings satisfying generalized contractive condition of integral type, Fixed Point Theory Appl. 2007 (2007), Article ID 54101, 9 pages.

[3] _ Common fixed point theorems for hybrid pairs of occasionally weakly compatible mappings defined on symmetric spaces, Panamer. Math. J. 18 (2008), no. 1, 55-62.

[4] _ Common fixed point theorems for occasionally weakly compatible mappings satisfying a generalized contractive condition, Math. Commun. 13 (2008), no. 2, 295-301.

[5] A. Aliouche and V. Popa, Common fixed point theorems for occasionally weakly compatible mappings via implicit relations, Filomat 22 (2008), no. 2, 99-107.

[6] _ General common fixed point theorems for occasionally weakly compatible hybrid mappings and applications, Novi Sad J. Math. 39 (2009), no. 1, 89-109.

[7] M. A. Al-Thagafi and N. Shahzad, Generalized I-nonexpansive selfmaps and invariant approximations, Acta Math. Sinica 24 (2008), no. 5, 867-876.

[8] _ A note on occasionally weakly compatible maps, Int. J. Math. Anal. (Ruse) 3 (2009), no. 1-4, 55-58.

[9] I. Altun and D. Turkoglu, Some fixed point theorems on fuzzy metric spaces with implicit relations, Commun. Korean Math. Soc. 23 (2008), no. 1, 111-124.

[10] A. Bhatt, H. Chandra, and D. R. Sahu, Common fixed point theorems for occasionally weakly compatible mappings under relaxed conditions, Nonlinear Anal. 73 (2010), no. 1, 176-182.

[11] H. Bouhadjera, A general common fixed point theorem for occasionally weakly compatible maps, An. Univ. Oradea, Fasc. Mat. 17 (2010), no. 2, 17-22.

[12] H. Bouhadjera and A. Djoudi, Common fixed point theorems of Greguš type for occasionally weakly compatible maps satisfying contractive conditions of integral type, An. Univ. Oradea Fasc. Mat. 16 (2009), 145-152.

[13] H. Bouhadjera, A. Djoudi, and B. Fisher, A unique common fixed point theorem for occasionally weakly compatible maps, Surv. Math. Appl. 3 (2008), 177-182.

[14] _ A unique common fixed point theorem for occasionally weakly compatible maps, Bull. Allahabad Math. Soc. 24 (2009), no. 1, 1-6.

[15] H. Bouhadjera and C. Godet-Thobie, Common fixed point theorems for occasionally weakly compatible single and set-valued maps, HAL-00273238, Version 1, 15 April 2008.

[16] Common fixed point theorems for occasionally weakly compatible maps, arXiv: $0812.3734 \mathrm{v} 2$ [math. FA], 17 June 2009.

[17] H. Chandra and A. Bhatt, Fixed point theorems for occasionally weakly compatible maps in probabilistic semi-metric space, Int. J. Math. Anal. (Ruse) 3 (2009), no. 9-12, 563-570.

[18] S. S. Chang, Y. J. Cho, and S. M. Kang, Nonlinear Operator Theory in Probabilistic Metric Spaces, Nova Science Publishers, Inc., New York, 2001.

[19] S. Chauhan, S. Kumar, and B. D. Pant, Common fixed point theorems for occasionally weakly compatible mappings in Menger spaces, J. Adv. Research Pure Math. 3 (2011), no. 4, 17-23.

[20] S. Chauhan and B. D. Pant, Common fixed point theorems for occasionally weakly compatible mappings using implicit relation, J. Indian Math. Soc. 77 (2010), no. (1-4), $13-21$.

[21] Lj. Ćirić, B. Samet, and C. Vetro, Common fixed point theorems for families of occasionally weakly compatible mappings, Math. Comp. Model. 53 (2011), 631-636.

[22] O. Hadžić and E. Pap, Fixed Point Theory in Probabilistic Metric Spaces, Dordrecht: Kluwer Academic publishers, 2001.

[23] M. Imdad, S. Kumar, and M. S. Khan, Remarks on some fixed point theorems satisfying implicit relations, Rad. Math. 11 (2002), no. 1, 135-143. 
[24] G. Jungck and B. E. Rhoades, Fixed points for set valued functions without continuity, Indian J. Pure Appl. Math. 29 (1998), no. 3, 227-238.

[25] _ Fixed point theorems for occasionally weakly compatible mappings, Fixed Point Theory 7 (2006), no. 2, 287-296.

[26] - Erratum: "Fixed point theorems for occasionally weakly compatible mappings" [Fixed Point Theory 7 (2006), no. 2, 287-296; MR2284600], Fixed Point Theory 9 (2008), no. 1, 383-384.

[27] M. A. Khan and Sumitra, Common fixed point theorems for occasionally weakly compatible maps in fuzzy metric spaces, Far East J. Math. Sci. 41 (2010), no. 2, 285-293.

[28] K. Menger, Statistical metrics, Proc. Nat. Acad. Sci. U.S.A. 28 (1942), 535-537.

[29] S. N. Mishra, Common fixed points of compatible mappings in PM-spaces, Math. Japon. 36 (1991), no. 2, 283-289.

[30] B. D. Pant and S. Chauhan, Common fixed point theorem for occasionally weakly compatible mappings in Menger space, Surv. Math. Appl. 6 (2011), 1-7.

[31] V. Popa, Some fixed point theorems for compatible mappings satisfying an implicit relation, Demonstratio Math. 32 (1999), no. 1, 157-163.

[32] _ A general coincidence theorem for compatible multivalued mappings satisfying an implicit relation, Demonstratio Math. 33 (2000), no. 1, 159-164.

[33] V. Popa and D. Turkoglu, Some fixed point theorems for hybrid contractions satisfying an implicit relation, Stud. Cercet. Ştinţ. Ser. Math. Univ. Bacau 1998 (1998), no. 8, 75-86.

[34] K. P. R. Sastry, G. A. Naidu, P. V. S. Prasad, V. M. Latha, and S. S. A. Sastry, A critical look at fixed point theorems for occasionally weakly compatible maps in probabilistic semi-metric spaces, Int. J. Math. Anal. (Ruse) 4 (2010), no. 27, 1341-1348.

[35] B. Schweizer and A. Sklar, Statistical metric spaces, Pacific J. Math. 10 (1960), 313-334.

[36] _ Probabilistic Metric Spaces, Elsevier, North Holland, New York, 1983.

[37] V. M. Sehgal and A. T. Bharucha-Reid, Fixed points of contraction mappings on probabilistic metric spaces, Math. Systems Theory 6 (1972), 97-102.

[38] S. Sharma and B. Deshpande, On compatible mappings satisfying an implicit relation in common fixed point consideration, Tamkang J. Math. 33 (2002), no. 3, 245-252.

[39] B. Singh and S. Jain, A fixed point theorem in Menger space through weak compatibility, J. Math. Anal. Appl. 301 (2005), no. 2, 439-448.

[40] C. Vetro, Some fixed point theorems for occasionally weakly compatible mappings in probabilistic semi-metric spaces, Int. J. Mod. Math. 4 (2009), no. 3, 277-284.

BADRI DATT PANT

Government Degree College

Champawat, 262523, Uttarakhand, India

E-mail address: badridatt.pant@gmail.com

SUNNY CHAUHAN

R. H. Government Postgraduate College

KASHIPUR, 244713, (U.S. NAGAR), UTtARAKHAND, INDIA

E-mail address: sun.gkv@gmail.com 